\title{
MicroRNA-382-5p inhibits osteosarcoma development and progression by negatively regulating VEZF1 expression
}

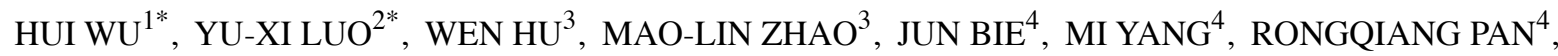 \\ NAN-XIANG HUANG ${ }^{5}$, GANG FENG $^{6}, \mathrm{KANG} \mathrm{LIU}^{6}$ and GUIQIN SONG ${ }^{7}$
}

\begin{abstract}
${ }^{1}$ Department of Orthopedics, Nanchong Central Hospital, The Second Clinical Institute of North Sichuan Medical College, Nanchong, Sichuan 637000; ${ }^{2}$ The First Clinical College, Anhui Medical University, Hefei, Anhui 230032;

${ }^{3}$ School of Medical Imaging, North Sichuan Medical College; ${ }^{4}$ Oncology Department; ${ }^{5}$ Department of Pediatric Surgery,

${ }^{6}$ Institute of Tissue Engineering and Stem Cells, Nanchong Central Hospital, The Second Clinical Institute of North Sichuan Medical College; ${ }^{7}$ School of Basic Medicine, North Sichuan Medical College, Nanchong, Sichuan 637000, P.R. China
\end{abstract}

Received September 27, 2020; Accepted May 12, 2021

DOI: 10.3892/ol.2021.13013

\begin{abstract}
Human osteosarcoma is the most frequent malignant primary bone tumor that mainly occurs in young adults and children. MicroRNAs (miRNAs/miRs) are abnormally expressed in human osteosarcoma and contribute to osteosarcoma initiation and development. The present study aimed to investigate the role of miR-382-5p in the nosogenesis of osteosarcoma and to identify a novel target for osteosarcoma treatment. miR-382-5p expression was detected in human osteosarcoma clinical tissues and cell lines, including 143B, U2OS and MG63, via reverse transcription-quantitative PCR analysis. Multiple bioinformatic prediction toowe used to identify the potential target genes of miR-382-5p and vascular endothelial zinc finger 1 (VEZF1), which were validated via the dual-luciferase reporter assay. MG63 and U2OS cells were transfected with miR-382-5p mimics. The Cell Counting Kit-8 assay was performed to assess cell proliferation, while the Transwell assay was performed to assess migration and invasion. Cell colony formation was measured via crystal violet
\end{abstract}

Correspondence to: Dr Kang Liu, Institute of Tissue Engineering and Stem Cells, Nanchong Central Hospital, The Second Clinical Institute of North Sichuan Medical College, 97 Renmin South Road, Nanchong, Sichuan 637000, P.R. China

E-mail: liukang@nsmc.edu.cn

Dr Guiqin Song, School of Basic Medicine, North Sichuan Medical College, 97 Renmin South Road, Nanchong, Sichuan 637000, P.R. China

E-mail: songguiqin@nsmc.edu.cn

*Contributed equally

Abbreviations: miRNAs/miRs, microRNAs; UTR, untranslated region

Key words: miR-382-5p, vascular endothelial zinc finger 1, osteosarcoma, proliferation, migration, invasion, apoptosis staining, and apoptosis was assessed via Annexin V/propidium iodide staining. The wound healing assay was performed to assess the migratory ability of U2OS and MG63 cells. Antitumor effects of miR-382-5p were evaluated in nude mice xenografts using U2OS cells. The results demonstrated that miR-382-5p expression was markedly downregulated in human osteosarcoma tissues and cell lines compared with adjacent normal tissues. Transfection of miR-382-5p mimics into MG63 and U2OS cells significantly inhibited the malignant behaviors of cells, including decreased proliferation, migration, diminished colony formation and invasion, and promoted osteosarcoma cell apoptosis. Bioinformatics prediction indicated that VEZF1 is a direct target gene of miR-382-5p. Overexpression of VEZF1 restored osteosarcoma tumor development inhibited by miR-382-5p in vivo. In addition, overexpression of miR-382-5p restrained the growth of xenograft osteosarcoma in nude mice following co-transfection, and overexpression of VEZF1 attenuated the inhibitory effect of miR-382-5p in nude mice. miR-382-5p acted as a tumor suppressor gene and inhibited the malignant biological behaviors of human osteosarcoma cells and functions associated with directly targeting VEZF1. Taken together, these results suggest that the miR-382-5p/VEZF1 interaction has an important role in osteosarcoma development and progression, and thus may be used as a diagnostic and therapeutic target for osteosarcoma.

\section{Introduction}

Human osteosarcoma is the most frequent malignant bone tumor, and its characteristics of malignant proliferation and invasion have made it the leading cause of cancer-associated mortality among teenagers and children (1-3). The incidence of osteosarcoma is approximately $4-5$ cases per million, which accounts for $>60 \%$ of all bone malignancies and $20 \%$ of all primary osteosarcomas in children $>5$ years old (4). Surgery and chemotherapy are the most common treatment methods for osteosarcoma (5). With improvements in therapeutic technologies, the 5-year survival rate of newly diagnosed patients with osteosarcoma without metastasis has improved 
to $\sim 60-70 \%$ (6). However, the 5-year survival rate of patients with osteosarcoma is only $20 \%$ because of the frequency of recurrent or metastatic disease (7). Thus, it is important to understand the molecular mechanisms underlying the initiation, progression, invasion and recurrence of osteosarcoma to identify novel therapeutic targets and develop rational strategies for clinical treatment of osteosarcoma.

MicroRNAs (miRNAs/miRs) are small (17-25 nucleotides) non-coding RNAs (8). These molecules are highly conserved endogenous RNAs that have an important role in post-transcriptional regulation of mRNAs for translation, and influence diverse biological activities (9). Although only the biological functions of a few miRNAs have been elucidated, miRNAs have been associated with osteosarcoma initiation, progression and invasion $(10,11)$. Increasing evidence suggest that miRNAs may perform gene regulation in different types of cancer, such as direct regulation of target genes, including oncogenes or tumor suppressor genes (12). For example, miR-142-3p has been demonstrated to inhibit osteosarcoma cell proliferation by targeting Rac1 (13). In addition, miR-142-3p has been reported to be highly overexpressed in osteosarcoma cells, to have pivotal roles in cellular invasion, proliferation, migration and apoptosis, and to induce E-cadherin expression and decrease expression of matrix metalloproteinase (MMP)2 and MMP9 (13). Thus, miRNAs are considered potential biomarkers and therapeutic targets for osteosarcoma.

Increasing evidence suggest that miR-382-5p is enriched in the serum of patients with ischemic stroke (14). Notably, serum miR-382-5p may be used as a non-invasive biomarker for potential diagnosis of ischemic stroke (15). In addition, aberrant miR-382-5p expression is frequently observed in different types of human cancer, including oral, breast cancer, ovarian, prostate, colorectal, lung and glioblastoma (16-19). However, the underlying molecular mechanisms and functional role of miR-382-5p in osteosarcoma tissues and cell lines remain largely unclear.

The present study aimed to investigate the biological effects of miR-382-5p on osteosarcoma cell migration, invasion, proliferation and apoptosis, both in vitro and in vivo. The results demonstrated that miR-382-5p expression was markedly downregulated in human osteosarcoma tumor tissues and cell lines, including MG63 and U2OS cells. In addition, overexpression of miR-382-5p inhibited malignant biological behaviors, including colony formation, proliferation, invasion and migration of osteosarcoma cell lines. Further studies demonstrated that miR-382-5p suppressed osteosarcoma development and progression by targeting VEZF1, and its aberrant expression remarkably reversed the antitumor effects of miR-382-5p overexpression in human osteosarcoma cell lines in vitro. Both the in vivo and in vitro experiments provide novel insights into the molecular functions of miR-382-5p in human osteosarcoma, which may be used as a potential therapeutic target for patients with osteosarcoma.

\section{Materials and methods}

Clinical samples and cell culture. A total of 20 paired (13 men and seven women; age range, 22-48 years; mean age, 33.8 years) adjacent normal tissues (within $5 \mathrm{~mm}$ from the tumor boundary) and osteosarcoma tumor tissues were collected from patients who underwent surgical resection at Nanchong Central Hospital (Nanchong, China) between January 2018 and December 2019. Resected tissues were immediately frozen in liquid nitrogen and stored at $-80^{\circ} \mathrm{C}$ until subsequent experimentation. The present study was approved by the Institutional Center Ethics Review Committee of Nanchong Central Hospital (Nanchong, China; approval no. 2020-002) and written informed consent was provided by all patients or their guardians prior to the study start.

Human osteosarcoma cell lines, including HFOB, HOBC, 143B and U2OS were purchased from The Cell Bank of Type Culture Collection of the Chinese Academy of Sciences, while MG63 cells were purchased from the American Type Culture Collection (ATCC, cat. no. ATCC ${ }^{\circledast}$ CRL-1690 ${ }^{\mathrm{TM}}$ ) and U2 osteosarcoma (ATCC ${ }^{\circledR}$ CRL-2611 ${ }^{\mathrm{TM}}$ ) were purchased from the indicated vendors and maintained in Dulbecco's Modified Eagle's Medium (DMEM, Gibco; Thermo Fisher Scientific, Inc.) supplemented with $10 \%$ fetal bovine serum (FBS; AccuRef Scientific, https://www.accurefsci.com), $100 \mathrm{IU} / \mathrm{ml}$ penicillin and $100 \mu \mathrm{g} / \mathrm{ml}$ streptomycin (all from Thermo Fisher Scientific, Inc.), at $37^{\circ} \mathrm{C}$ with $5 \% \mathrm{CO}_{2}$.

Transfection of miRNA mimics and plasmids. Human miR-382-5p mimics (50 nM, 5'-GAAGUUGUUCGUGGU GGAUUGG-3'), antisense or negative controls $(50 \mathrm{nM}$, NC-mimic; 5'-CTCGCTTCGGCAGCACA-3') were purchased from Shanghai GenePharma Co., Ltd.. The DNA coding sequence for VEZF1 was cloned into the pcDNA ${ }^{\mathrm{TM}} 3.1(+)$ expression vector (Thermo Fisher Scientific, Inc.), and the empty vector pcDNA3.1 was used as the NC. Osteosarcoma cell lines (MG63 and U2OS) in logarithmic growth phase were maintained in RPMI-1640 medium (Gibco; Thermo Fisher Scientific, Inc.) supplemented with $10 \%$ FBS. Cells were incubated at $37^{\circ} \mathrm{C}$ with $5 \% \mathrm{CO}_{2}$ in a sterility incubator and cultured for $24 \mathrm{~h}$. Cells were transfected with miR-382-5p mimics using the Lipofectamine ${ }^{\circledR} 3000$ kit (Thermo Fisher Scientific, Inc.), according to the manufacturer's protocol. Cells were collected for subsequent experimentation $48 \mathrm{~h}$ post-transfection.

Lentivirus packaging and infection. The 2nd lentiviral system purchased from Shanghai Genechem Co., Ltd., was used for lentivirus generation. Lentiviral particles were produced in Lenti-X $^{\mathrm{TM}} 293 \mathrm{~T}$ cells (Takara Bio Inc.; cat. no. 632180) in $10-\mathrm{cm}$ dish by transiently co-transfecting control lentiviral vector $(4.5 \mu \mathrm{g})$ or miR-382-5p overexpressing lentiviral vector $(4.5 \mu \mathrm{g})$ together with helper plasmids pHelper 1.0 (Gag and Pol; $2.5 \mu \mathrm{g}$ ) and pHelper 2.0 (VSVG; $2.0 \mu \mathrm{g}$ ) using the Lipofectamine $^{\circledast} 3000$ kit (Thermo Fisher Scientific, Inc.), according to the manufacturer's protocol. The vector constructions, verification by sequencing, virus packaging and collection of the corresponding viral supernatants were performed by Shanghai Genechem Co., Ltd. The lentivirus in medium of 293T cells were harvested by centrifuging at 75,000 $\mathrm{x}$ g for $1.5 \mathrm{~h}$ at $4^{\circ} \mathrm{C} 48 \mathrm{~h}$ post-transfection (Abcam), according to the manufacturer's protocol. Cells in the logarithmic growth phase were seeded into 96-well microplates (Costar; Corning, Inc.) at a density of $2 \times 10^{4}$ cells/well. Subsequently, the medium was replaced with DMEM supplemented with $10 \%$ FBS after overnight incubation at $37^{\circ} \mathrm{C}$. Cells were incubated for an 
additional 24,48, 72, and $96 \mathrm{~h}$, respectively. At each time point, $100 \mu \mathrm{l}$ CCK-8 reagent was added to each well and cells were incubated for an additional $2 \mathrm{~h}$ at $37^{\circ} \mathrm{C}$. Against a background control, the sample absorbance was measured at a wavelength of 490, using a microplate reader (Bio-Rad Laboratories, Inc.).

Apoptosis assay. MG63 and U2OS cells seeded into 6-well plates were transfected with the indicated miRNA mimics or the indicated plasmids and incubated at $37^{\circ} \mathrm{C}$ for an additional $24 \mathrm{~h}$. Cells were digested with $0.25 \%$ trypsin-EDTA solution (Sigma-Aldrich; Merck KGaA), terminated with cell medium, and harvested via centrifuged at $300 \mathrm{xg}$ for $5 \mathrm{~min}$ at $4^{\circ} \mathrm{C}$. After washing with PBS, the Annexin V-fluorescein Isothiocyanate (FITC)/PI Apoptosis kit (BD Biosciences) was used to detect cell apoptosis according to the manufacturer's protocols. Cells were washed three times with PBS and the binding buffer, and stained with Annexin V-FITC/PI for 15-20 min at room temperature in the dark. Cells were re-washed and the labeled cells were detected using a flow cytometer (CytoFLEX; Beckman Coulter, Inc.). Flow cytometry data were analyzed using Expo32 software (version 1.2; Beckman Coulter, Inc.).

Cell migration and invasion assays. Transwell ${ }^{\circledR}$ Boyden chambers (BD Biosciences) were used for in vitro cell migration assays, and chambers coated with or without Matrigel (BD Biosciences) were used to assess the migratory and invasive abilities of MG63 and U2OS cells in vitro. For pre-coating, Matrigel was maintained at $4^{\circ} \mathrm{C}$ overnight and the diluted with serum-free DMEM at dilution of 1:4 on ice. Subsequently, Matrigel was added to each upper chamber for coverage. Following incubation at $37^{\circ} \mathrm{C}$ for $1 \mathrm{~h}$, the chamber was rinsed with serum free DMEM followed by $50 \mu 1$ of DMEM supplemented with $10 \mathrm{~g} / 1 \mathrm{BSA}$ at $37^{\circ} \mathrm{C}$. Cells were resuspended in FBS-free fresh medium $48 \mathrm{~h}$ post-transfection. Cells were plated at a density of $1 \times 10^{5}$ cells/well into the upper chamber, and $200 \mu 1$ complete medium was loaded into the lower chamber. Following incubation at $37^{\circ} \mathrm{C}$ for $24 \mathrm{~h}$, the migratory and invasive cells on the lower surface of the membrane were fixed with $4 \%$ paraformaldehyde at $4^{\circ} \mathrm{C}$ for $1 \mathrm{~h}$ and stained with $0.1 \%$ crystal violet (Sigma-Aldrich; Merck KGaA) at room temperature for $15 \mathrm{~min}$, followed by a light microscopy (Nikon E600; Nikon Instrument Inc.) for visualization and photography. Stained cells were counted in five randomly selected fields at low-power (x100) magnification.

Dual-luciferase reporter assay. Given that miRNAs potentially interact with VEZF1, the binding sites between VEZF1 and miR-382-5p were predicted using IncBase version 2.0 (20). Candidate genes were targeted by miR-382-5p, and the binding sites of miR-382-5p were predicted using the online tools STarMirDB (http://sfold.wadsworth.org/starmirDB. php) and miRWalk (http://mirwalk.umm.uni-heidelberg.de). The predicted binding sites within the 3'-untranslated region (UTR) of VEZF1 mRNA (5'-AGGACAUUAAAUUGU ACAACUUU-3') and the corresponding mutated binding region (5'-AGGACAUUAAAUUGUCCUACCGU-3') were cloned into a luciferase-expressing vector pcDNA3.1. For the luciferase reporter assay, MG63 cells were transfected with different combinations of miR-382-5p mimics, control mimics, and pcDNA3.1-VEZF1 3'-UTR wild-type or mutant using the Lipofectamine ${ }^{\circledR} 3000$ kit (Thermo Fisher Scientific, Inc.), according to the manufacturer's protocol. Following transfection for $48 \mathrm{~h}$, cells were collected and luciferase activities were detected using the dual-luciferase reporter assay system (Promega Corporation) according to the manufacturer's instructions. Firefly luciferase activity was normalized to Renilla luciferase activity.

Reverse transcription-quantitative (RT-q)PCR. According to the manufacturer's protocols, total RNA and miRNA were extracted from osteosarcoma tissues and cell lines using TRIzol ${ }^{\circledR}$ reagent (AccuRef Scientific) and the miRNeasy mini kit (Qiagen GmbH), respectively. The PrimeScript ${ }^{\circledR}$ RT Master Mix Perfect Real-Time Reagent kit (Takara Bio,Inc.) was used to synthesize cDNA from total RNA, according to the manufacturer's instructions. For miRNA RT, the corresponding cDNA was synthesized using a universal tag (miScript II RT kit; Qiagen $\mathrm{GmbH}$ ). qPCR for miRNA and mRNA was subsequently performed using a standard protocol from the SYBR Green PCR kit (AccuRef Scientific) on an AB7500 RT-PCR instrument (Applied Biosystems; Thermo Fisher Scientific, Inc.) The following thermocycling conditions were used for qPCR: $95^{\circ} \mathrm{C}$ for $10 \mathrm{~min}$, and 40 cycles of $95^{\circ} \mathrm{C}$ for $10 \mathrm{sec}, 60^{\circ} \mathrm{C}$ for $30 \mathrm{sec}$ and $72^{\circ} \mathrm{C}$ for $10 \mathrm{sec}$. Relative quantification was determined by normalization to U6 or GAPDH. The following primer sequences were used for qPCR: VEZF1 forward, 5'-GCC TCAACTGACAGAGGAGAAG-3' and reverse, 5'-TCCAAA GTACTCCTTGGCTCC-3'; AKIRIN1 forward, 5'-GCACCT AGCTCTCCAGAACA-3' and reverse, 5'-CTTGTCCCATAC CGTCGCAT-3'; SLAIN1 forward, 5'-TTTTCCCCAGTGTTT TCGGAG-3' and reverse, 5'-CCCTGGAAGTGTAACCTT GCT-3'; LMTK3 forward, 5'-GTGTAATGTCTGCGTAAC CGC-3' and reverse, 5'-CCCCCAGTTGACAGAACTCC-3'; FOXN2 forward, 5'-GGGCTGGAATCTGCTGTTAGGG-3' and reverse, 5'-TCCATGGCTTCAACCAACTGT-3'; TOP1 forward, 5'-GTTCAAGCCACAGACCGAGA-3' and reverse, 5'-GCCTGGTAGAACGCTGACAA-3'; AMOTL2 forward, 5'-GGGTGATTCAGTTGGGTGCT-3' and reverse, 5'-GTA CCGTGGGTCAGTGACAG-3'; ARIH2 forward, 5'-ACT TGGGTGACATCTGCCTG-3' and reverse, 5'-GTCCTCTAT GTCCCCAGGGT-3'; DDX3X forward, 5'-GTAGCAGTC GTGGACGTTCT-3' and reverse, 5'-ACCTGTGTGCCAAGG TTTGA-3'; GAPDH forward, 5'-GTCTCCTCTGACTTC AACAGCG-3' and reverse, 5'-ACCACCCTGTTGCTGTAG CCAA-3'; miR-382-5p forward, 5'-ATCCGTGAAGTTGTT CGTGG-3' and reverse, 5'-TATGGTTGTAGAGGACTCCTT GAC-3'; and U6 forward, 5'-CGCTTCACGAATTTGCGT-3' and reverse, 5'-CTCGCTTCGGCAGCACA-3'. All experiments were performed in triplicate. PCR product specificity was confirmed via melting curve analysis. Relative expression levels were calculated using the $2^{-\Delta \Delta C q}$ method (21).

Western blotting. Protein samples from osteosarcoma tumor tissues and cell lines were isolated using RIPA lysis buffer (Sigma-Aldrich; Merck KGaA) and subsequently centrifuged at $12,000 \mathrm{x}$ g for $30 \mathrm{~min}$ at $4^{\circ} \mathrm{C}$. Total protein was quantified using the BCA method (Thermo Fisher Scientific, Inc.). Equal amounts of protein samples (20 $\mu \mathrm{g} / \mathrm{lane})$ were separated via $10 \%$ SDS-PAGE, transferred onto PVDF membranes and 
blocked with $5 \%$ skimmed milk at room temperature for $1 \mathrm{~h}$. The membranes were incubated with primary antibodies against VEZF1 (1:1,000; cat. no. SAB2102675; Sigma-Aldrich; Merck KGaA) and $\beta$-actin (1:10,000; cat. no. MFCD00164531; Sigma-Aldrich; Merck KGaA) at $4^{\circ} \mathrm{C}$ overnight. All primary antibodies were diluted in a Primary Dilution Solution (AccuRef Scientific) prior to incubation. Following the primary incubation, membranes were incubated with secondary HRP-conjugated polyclonal antibody anti-rabbit IgG (1:10,000; cat. no. MFCD00163923; Sigma-Aldrich; Merck KGaA) at room temperature for $1 \mathrm{~h}$. Protein bands were visualized using the Enhanced Chemiluminescence kit (Millipore, Sigma) and qualified using ImageJ software (version 1.49, National Institutes of Health).

Colony formation assay. Following trypsinization, MG63 and U2OS cells were suspended in medium that included $0.3 \%$ agar (low melt, Bio-Rad Laboratories, Inc.) and 10\% FBS (AccuRef Scientific), seeded into 12-well plates and subsequently transfected with the indicated miRNA mimics or the indicated plasmids followed by culturing for an additional $24 \mathrm{~h}$. Subsequently, 500 cells of each group were seeded into $35-\mathrm{mm}$ dishes, and the medium was replaced with fresh complete DMEM every 2 days. Following culturing for 14 days at $37^{\circ} \mathrm{C}$ in the incubator and washing three times with $\mathrm{PBS}$, the cells were fixed with $4 \%$ paraformaldehyde for $10 \mathrm{~min}$ at room temperature. Cells were subsequently stained with $0.1 \%$ crystal violet (Sigma-Aldrich; Merck KGaA) for $20 \mathrm{~min}$ at room temperature and washed with tap water. Cells were observed under a light microscope (Nikon E600; Nikon Instrument Inc.) with x200 magnification. All experiments were performed in triplicate.

Human osteosarcoma xenograft model. A total of 15 female BALB/c nude mice (4-6 weeks old and weighed 18-20 g) were obtained from Taconic Biosciences Company and acclimated in a specific-pathogen-free facility at the Experimental Animal Center of Nanchong Central Hospital, with a 12/12 h light/dark cycle, $40-60 \%$ humidity, $24-26^{\circ} \mathrm{C}$ temperature condition and free access to food and water for $\geq 1$ week. Stable NC lentivirus and stable miR-382-5p-expressing lentivirus-infected MG63 cells were resuspended in normal saline and mixed with an isopycnic of Matrigel ${ }^{\circledR}$ (BD Biosciences) at a final concentration of $5 \times 10^{6}$ cells $/ \mathrm{ml}$, respectively. Cells were injected into the left flank of nude mice subcutaneously at a density of $1 \times 10^{6}$ cells/mouse. Tumor size was measured every 7 days using vernier calipers. Following tumor cell inoculation, the mice were euthanatized at day 21 using $\mathrm{CO}_{2}$ inhalation with a flow rate of $3 \mathrm{l} / \mathrm{min}$ and euthanasia was confirmed via cardiac and respiration arrest. The tumors were subsequently isolated, weighed, imaged and subjected to subsequent analyses. All animal experiments were performed in accordance with the Guide for the Care and Use of Laboratory Animals (National Academies Press, 2011; Washington, USA). The animal experimental protocol was approved by the Ethical Review Committee for Animal Experiments of Nanchong Central Hospital (Nanchong, China; approval no. NSMC-2020-42), and all experiments were performed according to the AVMA guidelines.

Immunohistochemistry. While the tumors were pictured and weighed, partial tumor tissues were collected and cut into $5 \mathrm{~mm}^{3}$ size. Subsequently, tissues were fixed with $4 \%$ formaldehyde at $4^{\circ} \mathrm{C}$ more than $12 \mathrm{~h}$. Then, tissues were paraffin-embedded at $60^{\circ} \mathrm{C}$ overnight and cut into $5-\mu \mathrm{m}$ thick slices. Tumor tissues from xenograft mice were deparaffinized and rehydrated in an ethanol series. Antigen retrieval was performed according to previous experimental protocols (22). After washing with PBS and subsequent blocking with $3 \%$ hydrogen peroxide at room temperature for $10 \mathrm{~min}$, the sections were incubated with rabbit-anti-Ki-67 polyclonal antibody (1:400; cat. no. PA5-19462; Thermo Fisher Scientific, Inc.) overnight at $4^{\circ} \mathrm{C}$. Sections were washed three times with PBS, and the samples were incubated with HRP-conjugated secondary donkey-anti-rabbit monoclonal antibody (1:200; cat. no. ab6802; Abcam) at room temperature for $1 \mathrm{~h}$. Samples were re-washed five times with PBS and subsequently stained with $0.5 \%$ diaminobenzidine and counterstained with Mayer's hematoxylin at room temperature for 2-3 min. The mounted slices were observed under a light microscope (Nikon E600; Nikon Instrument Inc., magnification, x200).

Wound healing assay. U2OS and MG63 cells were seeded into 6-well plates at a density of $2 \times 10^{5}$ cells/well and allowed to adhere for $24 \mathrm{~h}$. Mimics were introduced into the cells and monolayer cells were scratched using a $200 \mu 1$ pipette tip after $24 \mathrm{~h}$. The medium was replaced with fresh serum-free RPMI-1640 medium. Wounded areas were observed at 0 and $48 \mathrm{~h}$ after culturing under a light microscope (Nikon E600; Nikon Instrument Inc., magnification, x100). The wound-healing rate (\%) was calculated using the following equation: Wound-healing rate $(\%)=($ width at $0 \mathrm{~h}$-width at $48 \mathrm{~h}) /$ width at $0 \mathrm{~h}) \mathrm{x} 100$. All experiments were performed in triplicate.

Statistical analysis. All quantitative data are presented as the mean \pm standard deviation. Normal distribution of all data were confirmed using the Kolmogorov-Smirnov D test, and differences between unpaired two groups were compared using unpaired two-tailed Student's t-test. Paired two-tailed Student's t-test was used to compare differences between adjacent tissues and tumor tissues. Comparisons among multiple groups were performed using ANOVA analysis. All experiments were performed in triplicate. SPSS v.19.0 software (IBM Corp.) was used to assess the correlation between miR-382-5p and VEZF1 mRNA expression, using Pearson's correlation analysis. $\mathrm{P}<0.05$ was considered to indicate a statistically significant difference.

\section{Results}

miR-382-5p expression is downregulated in osteosarcoma tumor tissues and cell lines. To investigate the role of miR-382-5p in osteosarcoma, RT-qPCR analysis was performed to detect miR-382-5p expression in 20 paired human osteosarcoma tissues and adjacent normal tissues. The results demonstrated that miR-382-5p expression was significantly downregulated in osteosarcoma tumor tissues compared with adjacent normal tissues ( $\mathrm{P}<0.01$; Fig. 1A). In addition, miR-382-5p expression was detected in human osteosarcoma cell lines. The results demonstrated that miR-382-5p expression was significantly downregulated in human osteosarcoma cell lines (143B, MG63 and U2OS) compared with the normal 
A

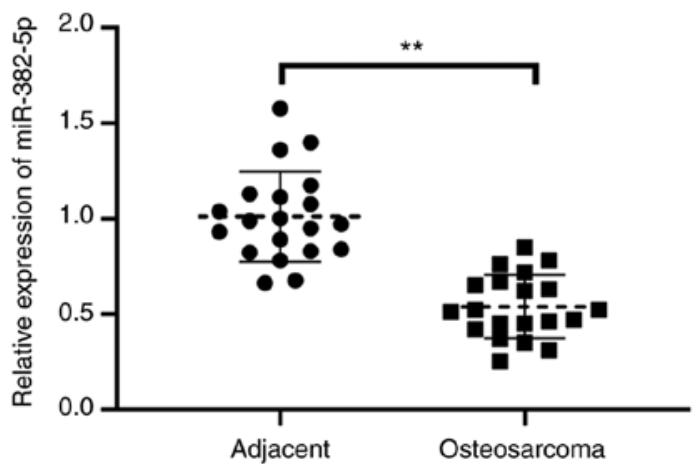

B

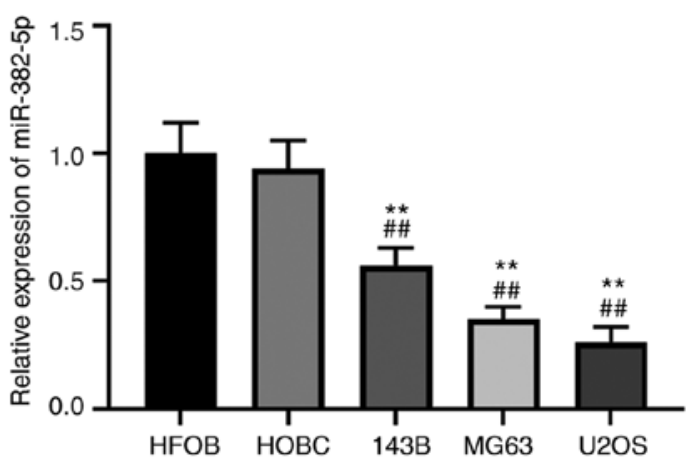

Figure 1. miR-382-5p expression is downregulated in osteosarcoma tissues and cell lines. (A) RT-qPCR analysis was performed to detect miR-382-5p expression in 20 paired osteosarcoma tissues and adjacent normal tissues. (B) RT-qPCR analysis was performed to detect miR-382-5p expression in normal cell lines (HFOB and HOBC) and three human osteosarcoma cell lines (143B, MG63 and U2OS). ${ }^{* *} \mathrm{P}<0.01$ vs. HFOB cells; ${ }^{\# \prime} \mathrm{P}<0.01$ vs. HOBC cells. miR, microRNA; RT-qPCR, reverse transcription-quantitative PCR.

HFOB and HOBC cell lines ( $\mathrm{P}<0.01$; Fig. 1B). Notably, MG63 and U2OS cells exhibited $\sim 80 \%$ reduction of miR-382-5p expression (Fig. 1B). Thus, these cell lines were selected for subsequent experimentation.

Overexpression of miR-382-5p inhibits proliferation, invasion and migration of osteosarcoma cells, and promotes apoptosis. According to the identifications in Fig. 1, downregulated miR-382-5p expression in human osteosarcoma tissues and osteosarcoma cell lines suggests that miR-382-5p may have important antitumor functions in the initiation and progression of osteosarcoma. Thus, the effects of miR-382-5p overexpression on the malignant behaviors of human osteosarcoma cells were investigated, including colony formation, proliferation, invasion and migration. MG63 and U2OS cells were transfected with miR-382-5p mimic or a NC miRNA mimic, and a 40-fold increase in miR-382-5p expression was observed at $48 \mathrm{~h}$ in miR-82-5p mimic-transfected MG63 and U2OS cells $(\mathrm{P}<0.01$; Fig. 2A). We then compared the proliferation of these cells by performing CCK-8 assays. In addition, overexpression of miR-382-5p significantly impeded the proliferation of both MG63 and U2OS cells (both $\mathrm{P}<0.01$; Fig. 2B). A nearly $50 \%$ decrease in $\mathrm{OD}_{450 \mathrm{~nm}}$ absorbance values was observed in miR-382-5p mimic-transfected MG63 and U2OS cells at $72 \mathrm{~h}$ after culturing. Furthermore, similar reductions in colony numbers were also observed in MG63 (P<0.01; Fig. 2C) and U2OS $(\mathrm{P}<0.01)$ cells with ectopic miR-382-5p expression, which suggests that miR-382-5p notably decreases the colony formation ability of human osteosarcoma cells. Notable reductions in the migratory $(\mathrm{P}<0.01$; Fig. $2 \mathrm{D})$ and invasive $(\mathrm{P}<0.01$; Fig. 2E) abilities of both MG63 and U2OS cells were also observed following transfection with miR-382-5p mimics. In addition, overexpression of miR-382-5p significantly elevated the apoptotic rates of MG63 and U2OS cells (both $\mathrm{P}<0.01$; Fig. 2F). Taken together, these results suggest that miR-382-5p exhibits tumor-suppressive functions in human osteosarcoma cells.

miR-382-5p directly targets VEZF1 in osteosarcoma cells. To further investigate the molecular mechanisms underlying the tumor-suppressive function of miR-382-5p in osteosarcoma, bioinformatics analysis was performed, using STarMirDB and miRWalk $(23,24)$, to predict the target genes based on the consensus binding sites of miR-382-5p. In the present study, the potential targets of miR-385-5p were predicted using ENCORI and TargetScan databases and the overlapped targets were screened using a Venn analysis (Fig. 3A). A total of nine overlapped targets of miR-382-5p were identified, and overexpression of miR-382-5p significantly inhibited the expression levels of VEZF1, FOXN2, and AMOTL2, particularly VEZF1 $(\mathrm{P}<0.01$; Fig. 3B). The dual-luciferase reporter assay was performed to determine whether VEZF1 is a direct target of miR-382-5p in osteosarcoma. The results demonstrated that luciferase activity significantly deceased following co-transfection of wild-type VEZF1 3'-UTR and miR-382-5p mimics but remained unchanged following co-transfection with mutated VEZF1 3'-UTR and miR-382-5p mimics or NC in MG63 cells $(\mathrm{P}<0.01$; Fig. 3C). These results suggest direct binding of miR-382-5p to the predicted 3'-UTR of VEZF1.

RT-qPCR and western blot analyses were performed to further verify the association between miR-382-5p and VEZF1 expression in osteosarcoma tissues and adjacent normal tissues, and the respective cell lines. The results demonstrated that the mRNA and protein expression levels of VEZF1 were significantly lower in adjacent normal tissues compared with osteosarcoma tissues $(\mathrm{P}<0.01$; Fig. 3D). Similarly, the mRNA and protein expression levels of VEZF1 were significantly lower in normal cells compared with osteosarcoma cells $(\mathrm{P}<0.01$; Fig. 3E). Furthermore, mRNA VEZF1 expression was inversely correlated with miR-382-5p expression in osteosarcoma tissues $(\mathrm{r}=-0.2182 ; \mathrm{P}<0.01$; Fig. 3F).

VEZF1 mRNA expression significantly decreased in MG63 cells transfected with miR-382-5p mimic compared with the control group $(\mathrm{P}<0.05$; Fig. 3G). Furthermore, western blot analysis demonstrated that VEZF1 protein expression significantly decreased in MG63 cells transfected with miR-382-5p mimic compared with the control group (Fig. $3 \mathrm{H}$ ). Collectively, these results suggested that VEZF1 is a direct downstream target of miR-382-5p in human osteosarcoma.

Overexpression of VEZF1 enhances cell proliferation, invasion and migration, while inhibiting apoptosis in osteosarcoma 
A

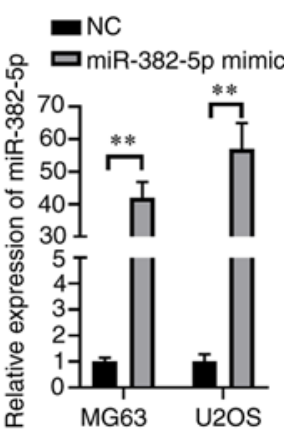

B

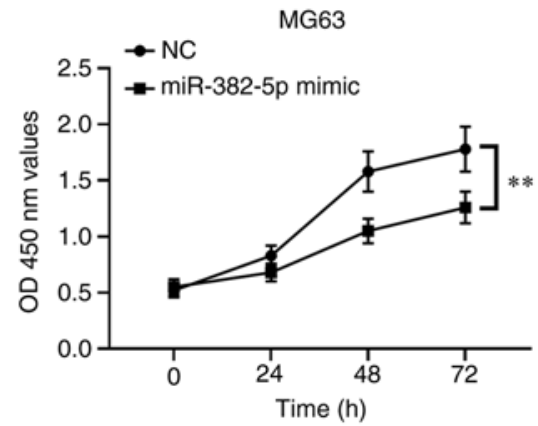

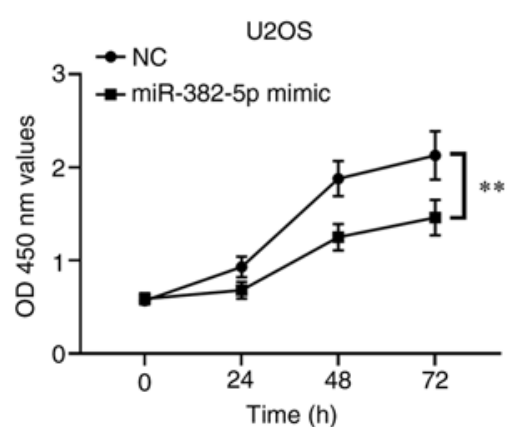
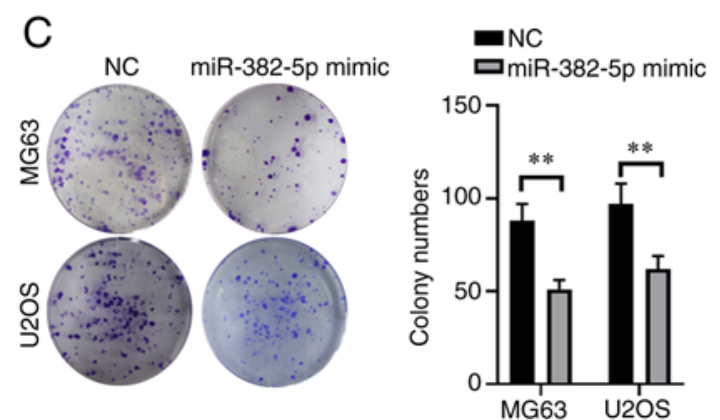

D

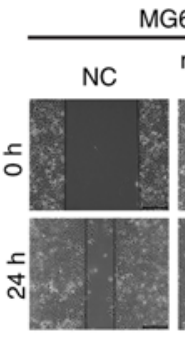

MG63

U2OS
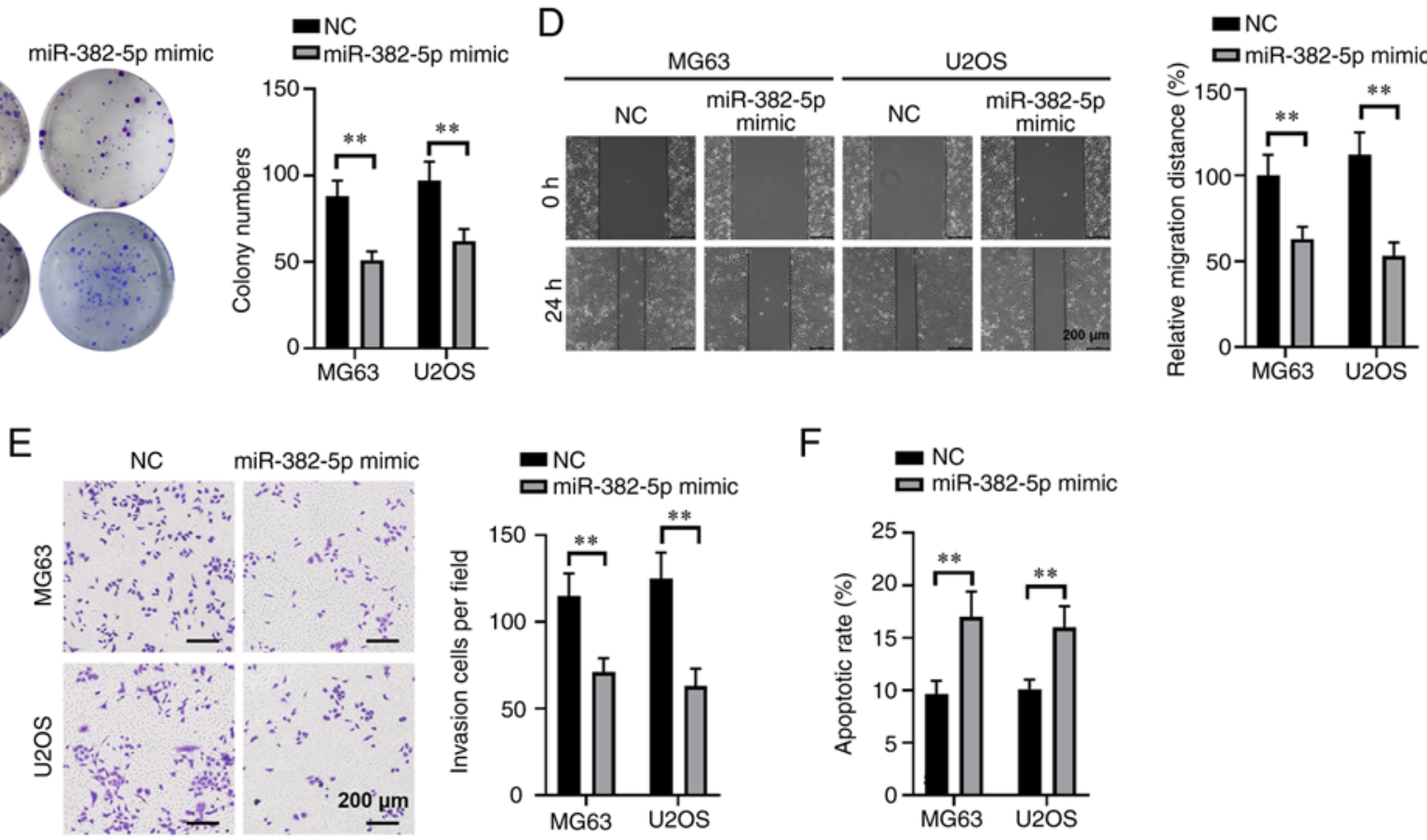

$\mathrm{F}$
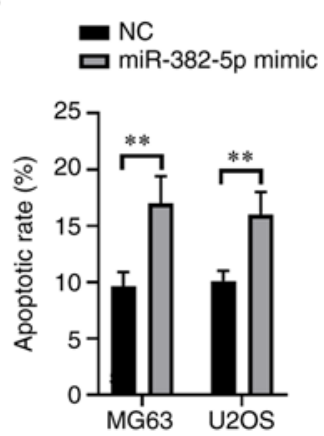

Figure 2. Overexpression of miR-382-5p inhibits cell proliferation, migration and invasion, while promoting apoptosis in osteosarcoma cells. (A) The efficiency of overexpression of miR-382-5p following transfection of MG63 and U2OS cells with NC mimic or miRNA-382-5p mimic was assessed via reverse transcription-quantitative PCR analysis. (B) The Cell Counting Kit-8 assay was performed to assess the proliferation of MG63 cells (left) and U2OS cells (right) transfected with NC mimic or miR-382-5p mimic. (C) The colony formation assay was performed to assess the number of MG63 and U2OS cell colonies formed following transfection with NC mimic or miR-382-5p mimic. (D) The wound healing assay was performed to assess the migratory ability of MG63 and U2OS cells transfected with NC mimic or miR-382-5p mimic. Scale bar, $200 \mu \mathrm{m}$. (E) The Transwell assay was performed to assess invasive ability of MG63 and U2OS cells transfected with NC mimic or miR-382-5p mimic. Scale bar, $200 \mu \mathrm{m}$. (F) Flow cytometric analysis was performed to detect apoptosis in MG63 and U2OS cells transfected with NC mimic or miR-382-5p mimic. ${ }^{* *} \mathrm{P}<0.01$. miR, microRNA; NC, negative control; OD, optical density.

cells. To determine whether VEZF1 promotes the malignant behaviors of osteosarcoma cell, including invasion, proliferation and migration in MG63 or U2OS cells, MG63 and U2OS cells were transfected with VEZF1-expressing plasmid to elevate VEZF1 protein expression ( $\mathrm{P}<0.01$; Fig. $4 \mathrm{~A})$. The results of the CCK- 8 assay demonstrated that overexpression of VEZF1 significantly enhanced the proliferation of MG63 and U2OS cells $(\mathrm{P}<0.05$; Fig. 4B). Furthermore, overexpression of VEZF1 significantly increased the colony formation ability of MG63 and U2OS cells ( $\mathrm{P}<0.01$; Fig. 4C). The results of the wound healing assay demonstrated that overexpression of VEZF1 promoted osteosarcoma cell migration in MG63 or U2OS cells compared with the NC group ( $\mathrm{P}<0.01$; Fig. 4D). Similarly, the results of the Transwell assay demonstrated that overexpression of VEZF1 promoted the invasive ability of MG63 and U2OS cells compared with the NC group $(\mathrm{P}<0.01$; Fig. 4E). Flow cytometric analysis demonstrated that overexpression of VEZF1 significantly decreased cell apoptosis compared with the control group $(\mathrm{P}<0.01$; Fig. $4 \mathrm{~F})$. Taken together, these results suggest that VEZF1 promotes malignant behaviors of MG63 and U2OS cells.

Overexpression of VEZF1 abrogates the effect of $\mathrm{miR}-382-5 \mathrm{p}$ on cell proliferation, invasion, migration and cell apoptosis. To further investigate the association between VEZF1 and miR-382-5p in human osteosarcoma cells, the effects of overexpressing VEZF1 on malignant behaviors of miR-382-5p mimic-transfected MG63 or U2OS cells were assessed. Western blot analysis demonstrated that overexpression of VEZF1 significantly increased VEZF1 expression in MG63 and U2OS cells compared with the miR-382-5p mimics group $(\mathrm{P}<0.01$; Fig. 5A). Similarly, the results of the CCK-8 assay demonstrated that overexpression of VEZF1 significantly enhanced the proliferation of MG63 or U2OS cells compared 
A

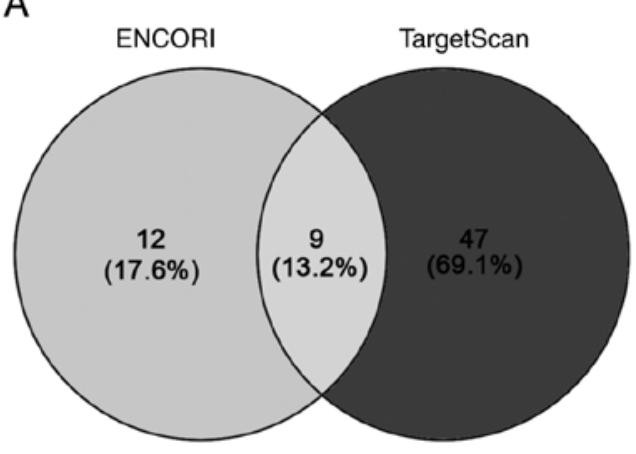

C
B $=\mathrm{NC}$

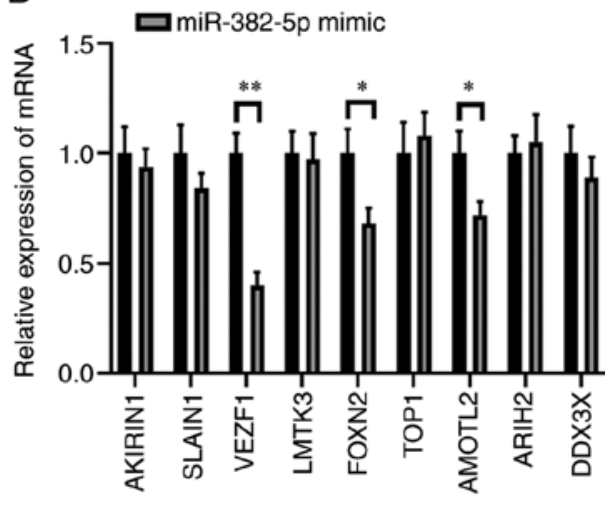

- NC

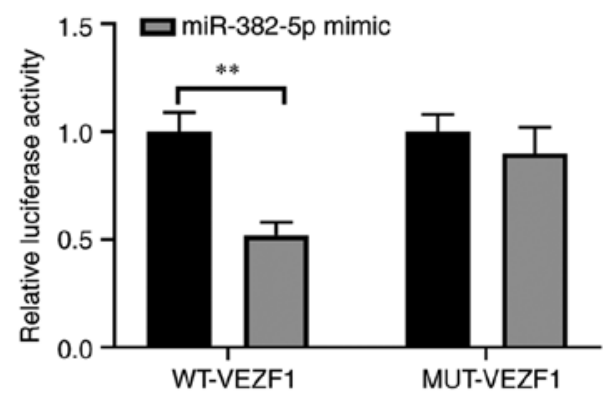

F
D

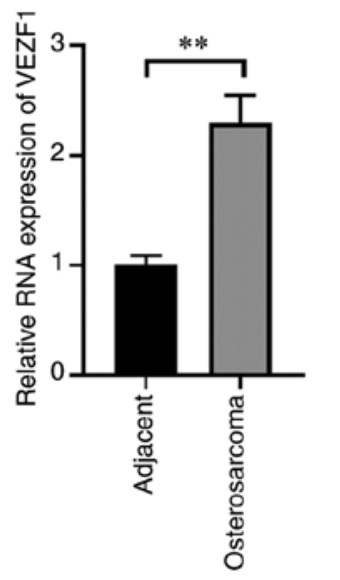

E
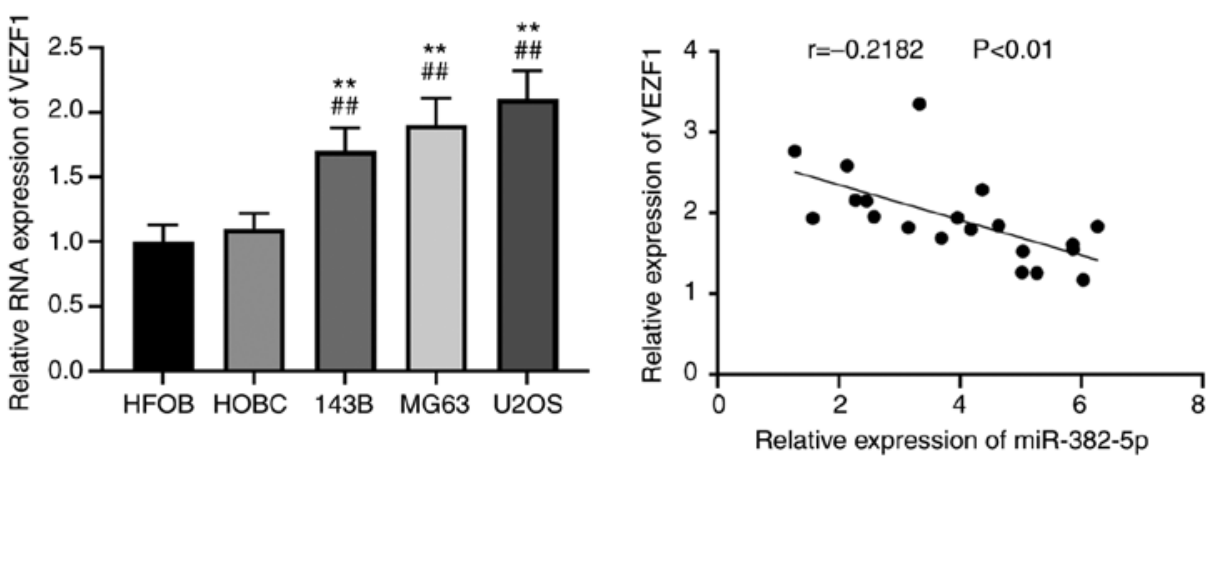

G
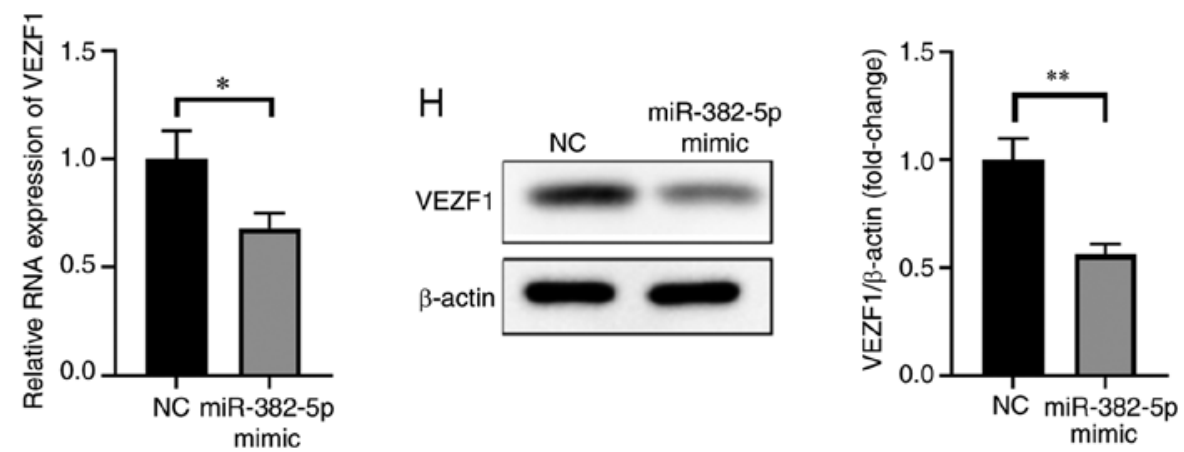

Figure 3. VEZF1 is a direct target of miR-382-5p in osteosarcoma. (A) Bioinformatics analysis was performed to identify the potential targets of miR-382-5p, among which nine mRNAs were selected for subsequent experimentations. (B) Relative expression of potential targets of miR-382-5p in MG63 cells following transfection with miR-382-5p mimic. (C) The mature miR-382-5p sequence, the putative binding sites of miR-382-5p and the corresponding wild-type and mutant sites of VEZF1 mRNA 3'-UTR (left) and the luciferase activity of the reporter vectors demonstrated that VEZF1 mRNA expression significantly decreased in MG63 cells following transfection with miR-382-5p mimics (right). (D) RT-qPCR analysis was performed to detect VEZF1 mRNA expression in osteosarcoma tissues and adjacent normal tissues. ${ }^{*} \mathrm{P}<0.05,{ }^{* * *} \mathrm{P}<0.01$. (E) RT-qPCR analysis was performed to detect VEZF1 mRNA expression in osteosarcoma cell lines (143B, MG63 and U2OS) and normal cell lines (HFOB and HOBC). ${ }^{* *} \mathrm{P}<0.01$ vs. HFOB cells; ${ }^{* \#} \mathrm{P}<0.01$ vs. HOBC cells. (F) Pearson's correlation analysis was performed to assess the correlation between miR-382-5p and VEZF1 expression in osteosarcoma. (G) RT-qPCR analysis was performed to detect VEZF1 mRNA expression in MG63 cells transfected with miR-382-5p mimic or NC mimic. "P<0.05. (H) Western blot analysis was performed to detect VEZF1 protein expression in cells transfected with miR-382-5p mimic or NC mimic. " $\mathrm{P}<0.01$. VEZF1, vascular endothelial zinc finger 1; miR, microRNA; UTR, untranslated; RT-qPCR, reverse transcription-quantitative PCR; NC, negative control; WT, wild-type; MUT, mutant. 
A
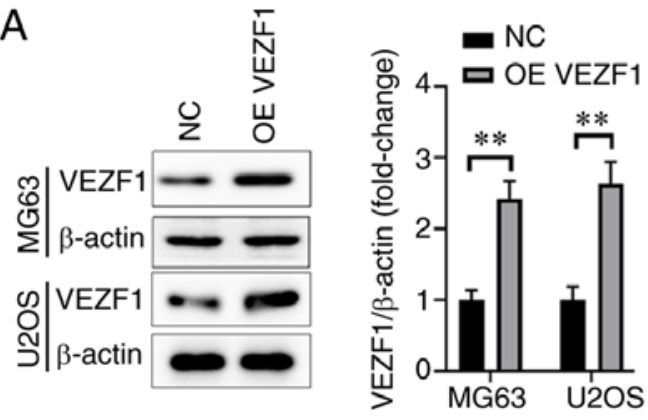

B

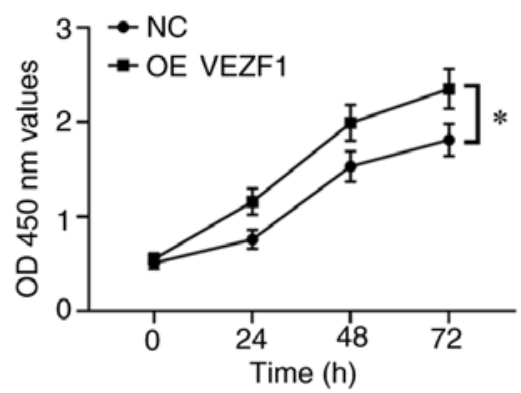

D

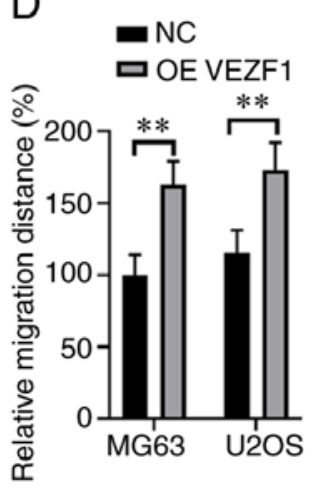

U2OS

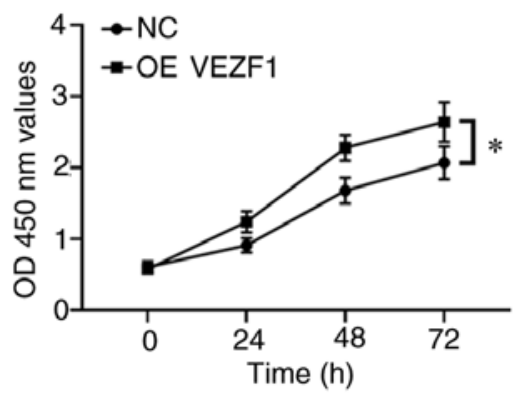

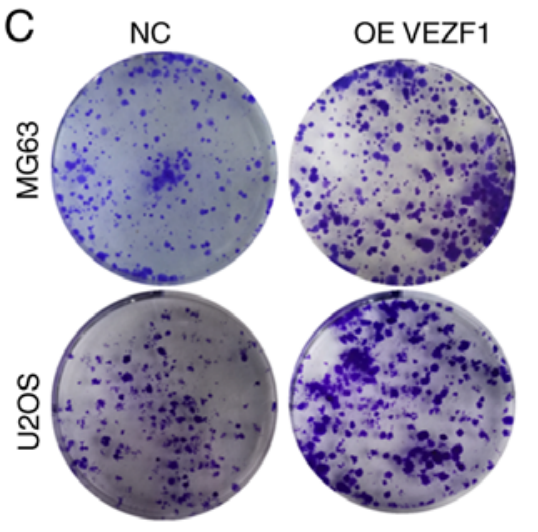

C
$=\mathrm{NC}$ 口OE VEZF1

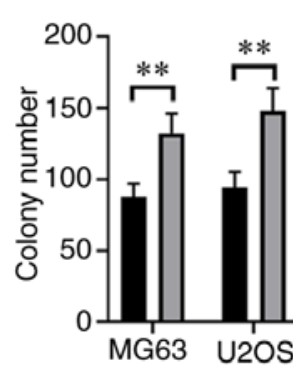

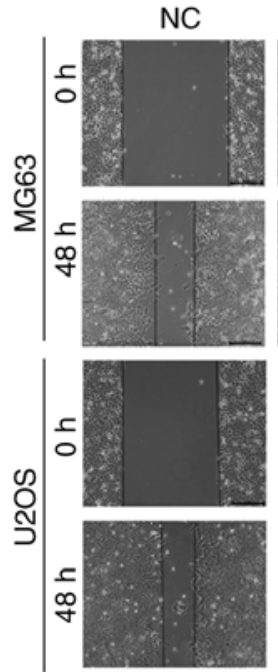

OE VEZF1
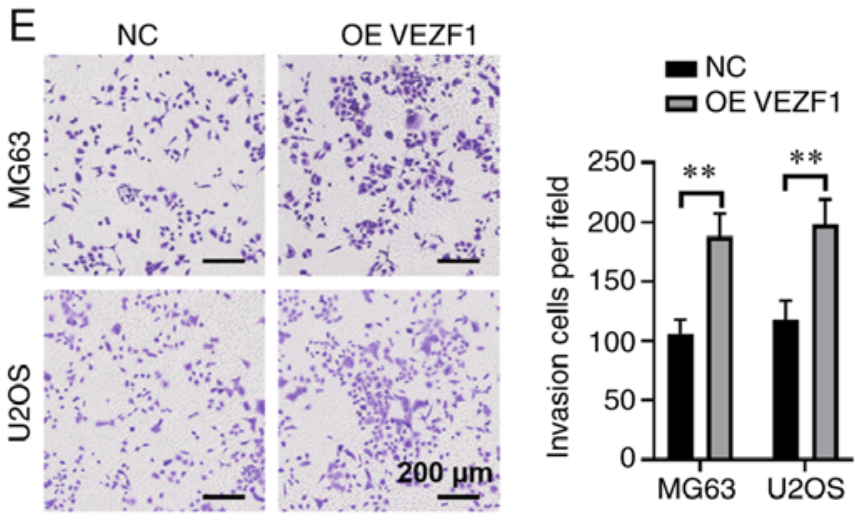

$\mathrm{F}$

$$
\text { - } \text { 으 } \mathrm{NEZF1}
$$

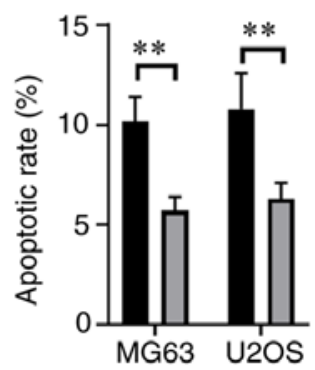

Figure 4. Overexpression of VEZF1 promotes cell proliferation, migration and invasion, while suppressing apoptosis in osteosarcoma cells. (A) Western blot analysis was performed to detect VEZF1 protein expression. (B) The Cell Counting Kit-8 assay was performed to assess the proliferation of MG63 (left) and U2OS (right) cells in the NC and overexpression VEZF1 groups. (C) Overexpression of VEZF1 promoted the proliferation of MG63 and U2OS cells as demonstrated by the cell colony formation assay. (D) Overexpression of VEZF1 promoted the migratory ability of MG63 and U2OS cells as demonstrated by the wound healing. Scale bar, $200 \mu \mathrm{m}$. (E) Overexpression of VEZF1 promoted the invasive ability of MG63 and U2OS cells as demonstrated by the Transwell assay. Scale bar, $200 \mu \mathrm{m}$. (F) Overexpression of VEZF1 significantly suppressed apoptosis of MG63 and U2OS cells. "P<0.05; ${ }^{* *} \mathrm{P}<0.01$. VEZF1, vascular endothelial zinc finger 1; $\mathrm{NC}$, negative control; $\mathrm{OE}$, overexpression; $\mathrm{OD}$, optical density.

with the miR-382-5p mimics group $(\mathrm{P}<0.01$; Fig. 5B). Furthermore, simultaneous overexpression of VEZF1 in miR-382-5p-transfected MG63 and U2OS cells significantly increased the number of cell colonies $(\mathrm{P}<0.01$; Fig. $5 \mathrm{C})$, and significantly induced the cell migratory $(\mathrm{P}<0.01 ;$ Fig. $5 \mathrm{D})$ and invasive $(\mathrm{P}<0.01$; Fig. 5E) abilities, while decreasing apoptosis $(\mathrm{P}<0.01 ;$ Fig. 5F). Taken together, these results suggested that simultaneous overexpression of VEZF1 reverses the effects of miR-382-5p overexpression on malignant behaviors of human osteosarcoma cells.
Overexpression of VEZF1 attenuates the inhibitory effect of miR-382-5p in an animal model. To further investigate the tumor-inhibiting function of miR-382-5p via VEZF1 in human osteosarcoma in vivo, empty lentivirus-infected control stable U2OS cells (LV), miR-382-5p-expressing lentivirus-infected stable U2OS cells (LV-miR-382-5p) and VEZF1 overexpression vectors (VEZF1) were inoculated and co-transfected into LV-miR-382-5p cells on the left flank of model mice via subcutaneous injection. Some of the mice were sacrificed at day 18 following tumor inoculation. The 
A

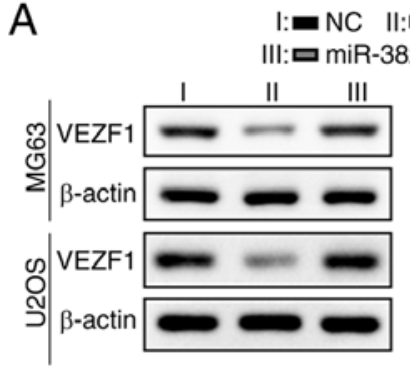

C

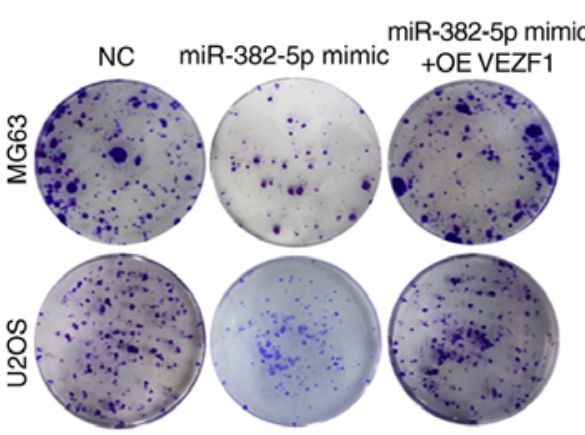

B

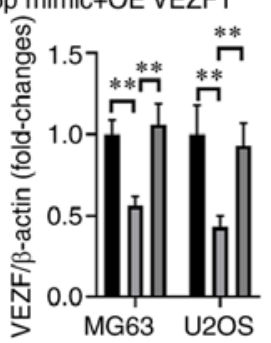

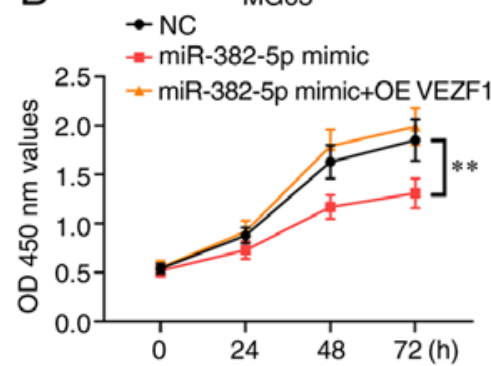

U2OS

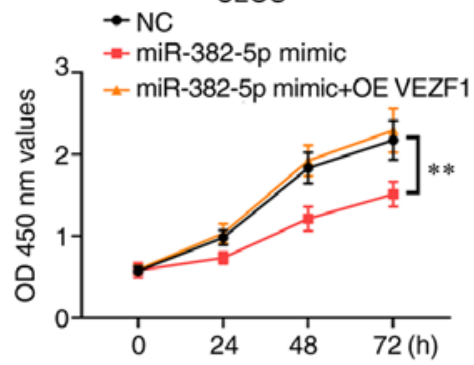

D NC $\quad \begin{gathered}\text { miR-382-5p miR-382-5p } \\ \text { mimic }\end{gathered}$ mimic+OE VEZF1
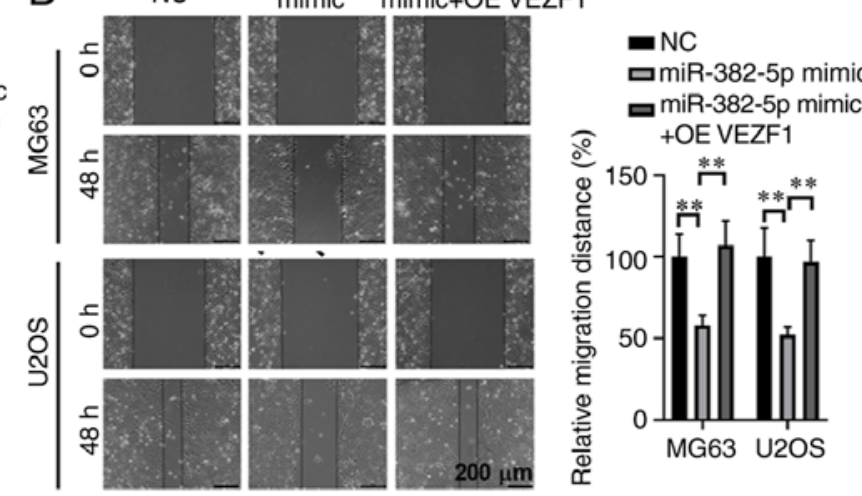

E

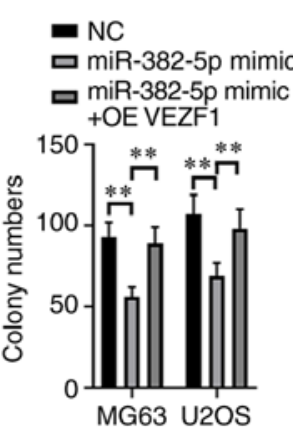

$\mathrm{F}$

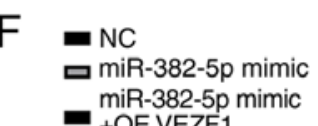

-OE VEZF1
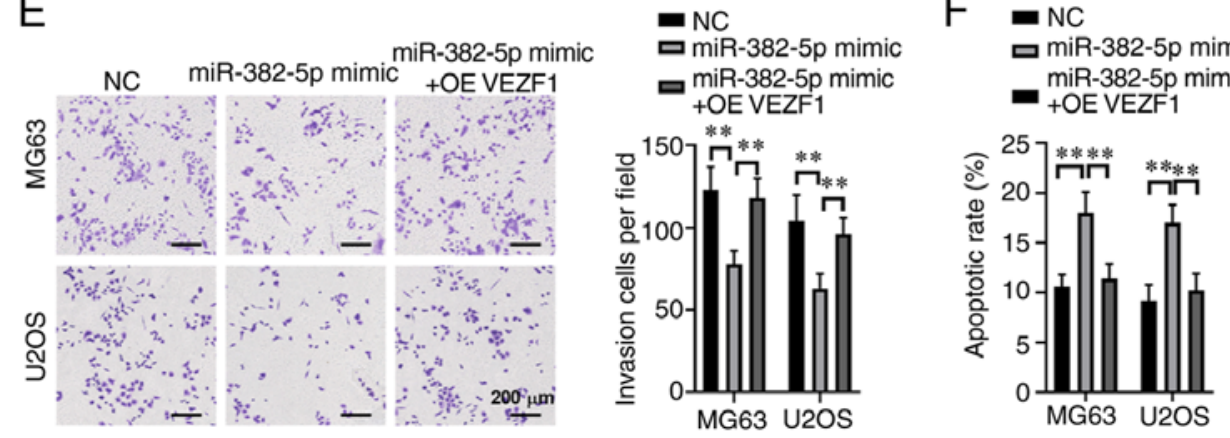

Figure 5. Simultaneous overexpression of VEZF1 reverses the antitumor effects of miR-382-5p mimic on malignant behaviors of human osteosarcoma cells (A) Western blot analysis was performed to detect VEZF1 protein expression following transfection of MG63 and U2OS cells with miRNA-382-5p mimics alone or the indicated miRNA-382-5p mimics and recombinant plasmids pcDNA-VEZF1 co-transfection and normal control. (B) The Cell Counting Kit-8 assay was performed to assess the proliferation of MG63 cells (left) and U2OS (right) cells following transfection with NC, miR-382-5p mimics alone or miR-382-5p mimics and recombinant plasmids pcDNA-VEZF1. (C) VEZF1 abrogated the regulatory effect of miR-382-5p on the proliferation of MG63 and U2OS cells as demonstrated by the cell colony formation assay. Scale bar, $200 \mu \mathrm{m}$. (D) VEZF1 abrogated the regulatory effect of miR-382-5p on the migratory ability of MG63 and U2OS cells as demonstrated by the wound healing assay. (E) VEZF1 abrogated the regulatory effect of miR-382-5p on the invasive ability of MG63 and U2OS cells as demonstrated by the Transwell assay. Scale bar, $200 \mu \mathrm{m}$. (F) Flow cytometric analysis was performed to detect apoptosis of MG63 and U2OS cells transfected with NC, miR-382-5p mimics alone or miR-382-5p mimics and recombinant plasmids pcDNA-VEZF1. ${ }^{* *} \mathrm{P}<0.01$. VEZF1, vascular endothelial zinc finger 1; miR, microRNA; NC, negative control; OE, overexpression; OD, optical density.

results demonstrated that 21 days after xenografting, the tumor volume significantly decreased in U2OS cells stably transfected with LV-miR-382-5p significantly. In addition, the weight of the mice decreased by $70 \%$ compared with the miR-382-5p mimics and VEZF1 co-transfection or miR-NC groups (Fig. 6A and B). RT-qPCR analysis demonstrated a notable increase in miR-382-5p expression following transduction with miR-382-5p lentivirus but had no obvious change after overexpressing VEZF1, whereas VEZF1 mRNA expression significantly decreased following transfection with miR-382-5p mimics but significantly increased after overexpressing VEZ1 $(\mathrm{P}<0.01$; Fig. 6C), which confirms the association between miR-382-5p and VEZF1 in human osteosarcoma.
Immunohistochemical analysis of $\mathrm{Ki}-67$ in tumor tissues indicated pleomorphic poorly differentiated neoplastic cells, confirming cancerous tissue growth (Fig. 6D). Subsequent experiments focused on miR-382-5p-mediated regulation of B-cell lymphoma 2 (Bcl2) and Bax, which are downstream of the vascular endothelial growth factor (VEGF) signaling pathway (25). Western blot analysis demonstrated that overexpression of miR-382-5p promoted Bax expression and suppressed Bcl2 expression, while simultaneously promoting cleaved caspase-3 expression ( $\mathrm{P}<0.01$; Fig. $6 \mathrm{E})$. In addition, overexpression of VEZF1 attenuated the suppression of miR-382-5p in osteosarcoma cells. Taken together, these results suggest that the size and weight of xenograft tumors in nude mice significantly decrease following transfection with miR-372-3p mimics. 
A

$\mathrm{I}: \rightarrow-\mathrm{NC} \quad \mathrm{II}:-\mathrm{miR}-382-5 \mathrm{p}$ mimic+OE VEZF1 III: -1- miR-382-5p mimic

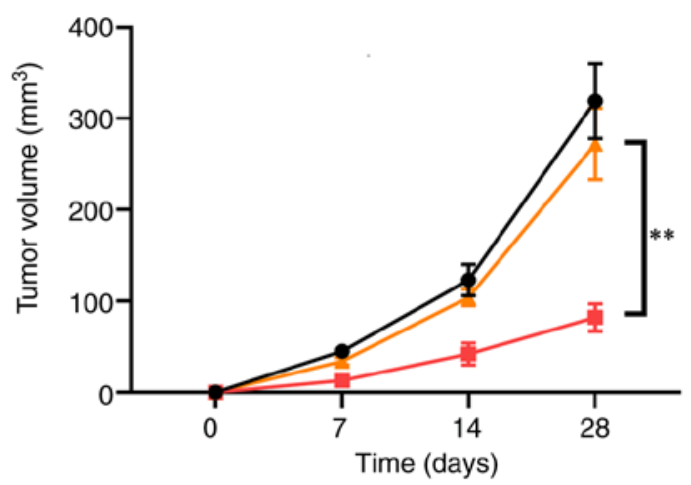

D

C

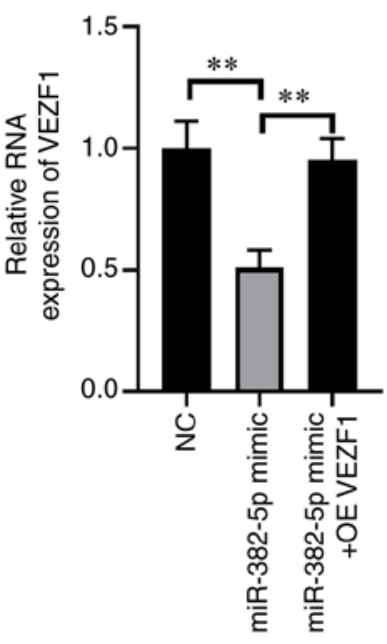

E
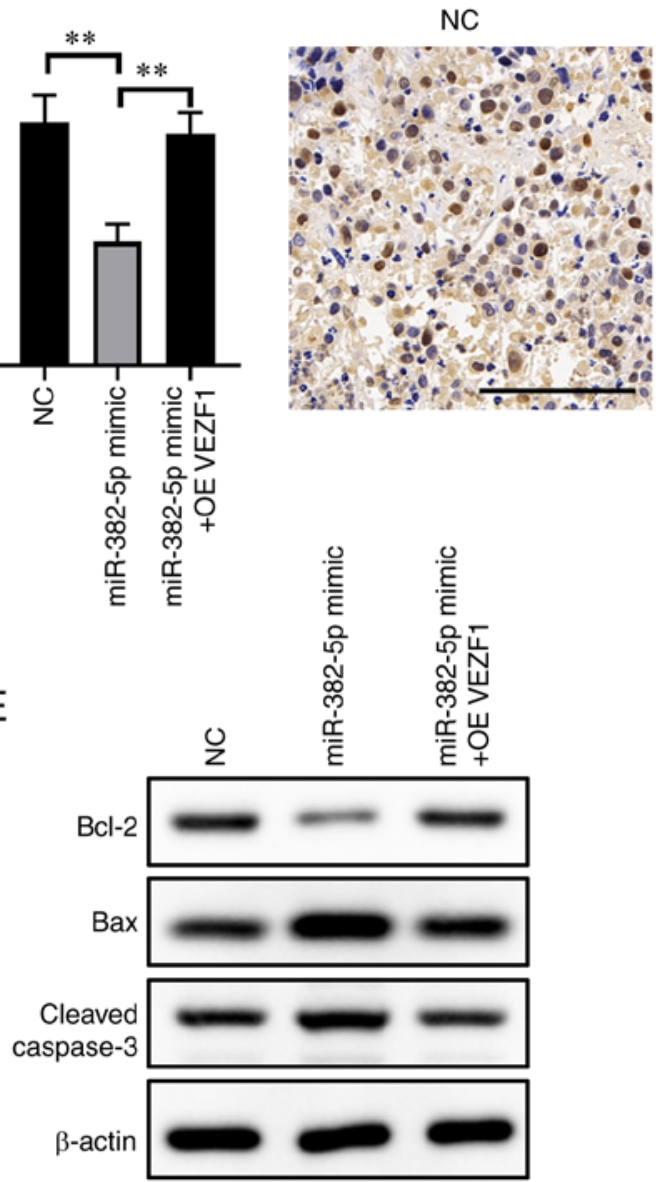

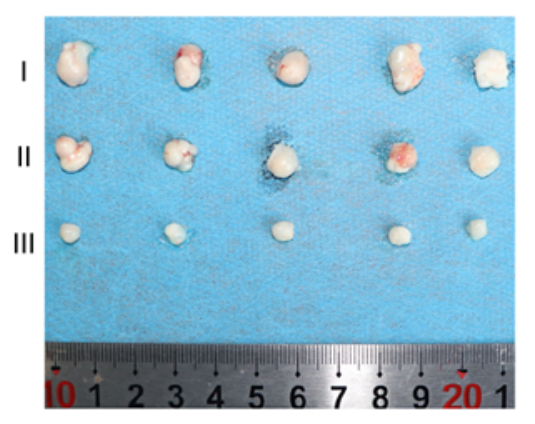

B

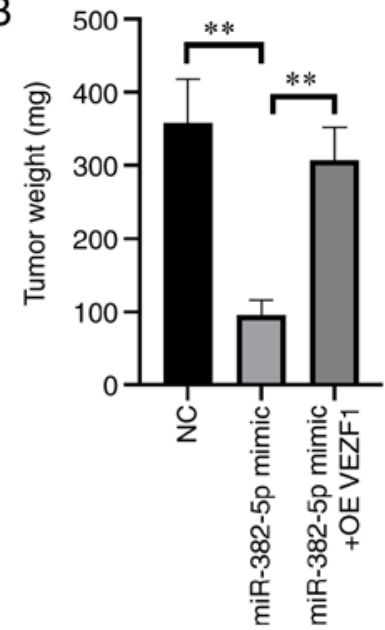

miR-382-5p mimic +OE VEZF1
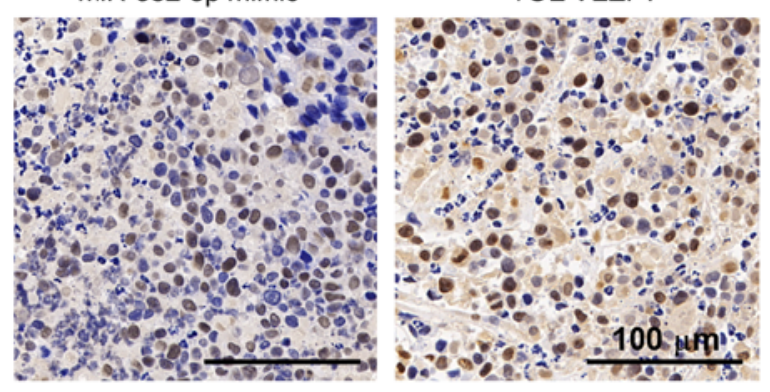

miR-382-5p mimic
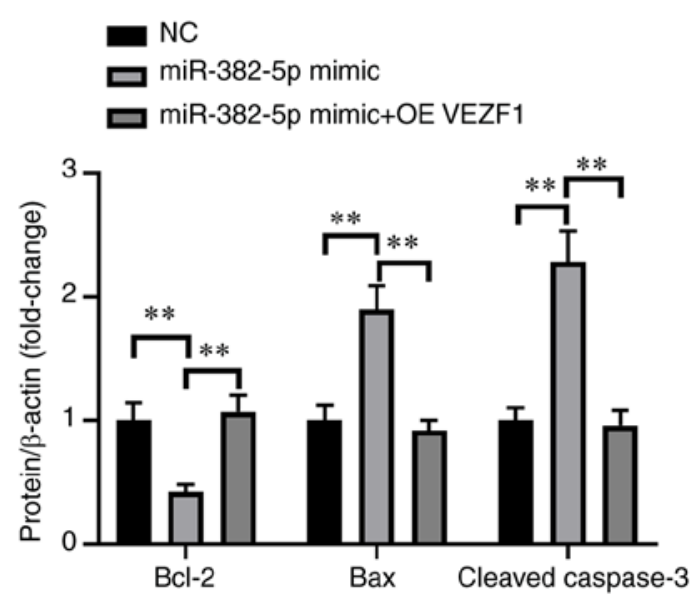

Figure 6. Overexpression of VEZF1 restores osteosarcoma tumor development inhibited by miR-382-5p in vivo. (A) The tumor growth curves during the observation period of 21 days. The xenograft nude mice model was constructed using U2OS cells. Tumor volumes were measured every 7 days. Stable U2OS cells transfected with miRNA-382-5p mimics, control cells or miRNA-382-5p overexpression and recombinant plasmids pcDNA-VEZF1 co-transfection

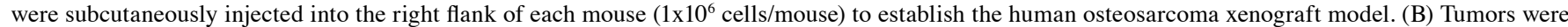
dissected 1 month after xenografting and weighed. (C) Reverse transcription-quantitative PCR analysis was performed to detect VEZF1 expression in the different groups. (D) Immunohistochemistry analysis was performed to detect Ki-67 expression in the different groups. Scale bar, $100 \mu \mathrm{m}$. (E) Western blot analysis was performed to detect the protein expression levels of Bc12, Bax and cleaved caspase- 3 in xenograft tumor tissues. ${ }^{* *} \mathrm{P}<0.01$. VEZF1, vascular endothelial zinc finger 1; miR, microRNA; Bcl2, B-cell lymphoma 2; NC, negative control; OE, overexpression.

\section{Discussion}

The first miRNAs, which are small non-coding RNA segments ( 22 nucleotides in length) were identified in 1993 (in Caenorhabditis elegans) (26). These miRNAs have diverse roles in cellular development, differentiation, apoptosis and metabolism, including osteoma initiation and normal osteogenesis (9). These molecules have important roles in various 
functions, such as those of tumor-suppressor genes or oncogenes, so when they are disordered, they contribute to initiation and progression of cancer (27). Recent studies have reported that aberrant expression of miRNAs has been demonstrated in a range of human diseases, including various malignancies (28-30). miR-382-5p has been repeatedly observed in various human malignancies, including lung, oral, ovarian, breast and prostate cancers (16-19). Given that miR-382-5p has been reported to be frequently and significantly reduced in tumor tissues and tumor-associated cell lines, it was speculated that miR-382-5p functions as a tumor-suppressor gene or oncogene in osteosarcoma. However, the highly complex molecular mechanisms underlying miR-382-5p inhibition of osteosarcoma cells remain unclear.

The results of the present study demonstrated that miR-382-5p expression was markedly downregulated in clinical osteosarcoma tumor tissues and osteosarcoma-associated cell lines compared with normal tissues and cell lines. In addition, miR-382-5p exerted antitumor functions in human osteosarcoma cells, including restraining cell colony formation, proliferation, migration and invasion, as well as enhancing apoptosis in vitro and suppressing tumor growth in vivo. These results are in accordance with previous findings, demonstrating that miR-382-5p expression is notably downregulated in cancer cells $(16,31,32)$. Previous study had demonstrated that miR-382-5p acts as tumor suppressor to inhibit the metastasis and relapse of osteosarcoma by targeting YB-1 (33), while circ_0001658 can significantly promote the proliferation and metastasis of osteosarcoma by modulating the miR-382-5p/YB-1 axis (34). A recent study also reported that LINC00265 can promote the proliferation, migration, invasion and angiogenesis of osteosarcoma by targeting miR-382-5p mediated SAT1 and VAV3 (35). In the present study, bioinformatics analysis was performed to identify a target gene of miR-382-5p as VEZF1, which was identified as a novel and functional target of miR-382-5p via the dual-luciferase reporter assay. Overexpression analyses of VEZF1 in osteosarcoma cells with ectopic expression of VEZF1 was also performed. The results demonstrated that overexpression of VEZF1 notably attenuated the tumor-suppressive role of miR-382-5p mimics on the malignant behaviors of human osteosarcoma cells. Taken together, these results suggest that the miR-382-5p-VEZF1 axis may act as a novel molecular target for developing therapeutics against osteosarcoma.

Osteosarcoma is the most common primary bone malignancy that predominantly occurs in children, adolescents and young adults (36). With improvements in technology, the 5-year overall survival rate has increased to $\sim 60-70 \%$ (37). However, the response of osteosarcoma to chemotherapy remains poor, and there is a risk of recurrence and metastasis following surgical excision and chemotherapy (38). Biomarkers are considered one of the most effective tools to determine the prognosis of a tumor following chemotherapy (39). A previous study demonstrated that miRNAs are significantly upregulated in serum samples from patients with breast cancer (40). However, due to the deficiency of serum samples, miR-382-5p expression was unable to be detected in serum samples of patients with osteosarcoma in the present study. miR-382-5p expression was only detected in tissue samples and the results demonstrated that miR-382-5p was significantly downregulated in clinical osteosarcoma tissues and osteosarcoma-associated cell lines. Despite the limitation in serum samples, the results of the present study and previous findings suggest that miR-382-5p may serve as a novel biomarker for the prognosis of patients with osteosarcoma. Considering the prognostic significance, serum miR-382-5p expression and its prognostic value in osteosarcoma will be investigated in prospective studies.

Recent studies have reported that miR-382-5p has important functions, including a tumor-suppressor or oncogene, in the context of different types of cancer. For examples, previous studies have demonstrated that miR-382-5p expression is downregulated in human ovarian tumor tissues and cancer-associated cell lines $(17,32)$, whereas miR-382-5p expression is upregulated in breast and glioma tumor tissues and cancer-associated cell lines $(16,31)$. However, the expression and function of miR-382-5p in osteosarcoma remains unclear. The results of the present study demonstrated that miR-382-5p expression was markedly downregulated in osteosarcoma tissues and cell lines, and significantly low expression levels were detected in tumor tissues. In vivo and in vitro overexpression of miR-382-5p inhibited proliferation, invasion and migration of osteosarcoma cells, and enhanced apoptosis. Taken together, these results suggest that overexpression of miR-382-5p exerts a tumor-suppressive effect in osteosarcoma.

A previous study reported that miRNAs recognize and combine specific target mRNAs depending on complete or partial base-pairing, mostly at the 3'-UTR of the target genes, to regulate gene expression following transcription, which includes both tumor suppressors and oncogenes (41). Bioinformatic analysis was performed in the present study to predict gene targets of miR-382-5p. The results revealed that VEZF1 has a highly conserved miR-382-5p binding sequence in the mRNA 3'-UTR. The results of the dual-luciferase reporter assay confirmed the direct association between miR-382-5p and VEZF1 in human osteosarcoma cells.

VEZF1 is a Krüppel-like zinc finger protein that plays a vital role in vascular development, and is a member of the VEGF signaling pathway (42). Abnormal expression of VEZF1 contributes to the pathogenesis of different disorders, including cancer and cardiovascular diseases (43). Recent study has reported that a small molecule inhibitor of VEZF1, Vec6, prevents wound healing and angiogenesis (44). Increasing evidences suggest that VEZF1 plays a major role in the development and progression of malignancy $(43,45,46)$. The results of the present study demonstrated that overexpression of VEZF1 in vitro and in a nude mice model increased proliferation, invasion and migration of osteosarcoma cells, and decreased apoptosis. Furthermore, overexpression of VEZF1 notably attenuated the tumor-suppressive effects of miR-382-5p mimics in human osteosarcoma cells and the animal model, which suggests that VEZF1 may act as an oncogene in human osteosarcoma cells. However, further studies are required to screen potential targets of miR-382-5p other than VEZF1 to determine the tumor-suppressive functions of miR-382-5p in human osteosarcoma.

In conclusion, the results of the present study demonstrated that miR-382-5p acts as a tumor-suppressor gene, whose ectopic expression inhibits the malignant biological behaviors of human osteosarcoma cells in vitro and in a nude mice model. VEZF1 was identified as a direct target gene of miR-382-5p, 
and its overexpression remarkably attenuated the inhibitory effect of miR-382-5p mimics in human osteosarcoma cells. The miR-382-5p/VEZF1 axis provides novel insights into the pathogenesis of osteosarcoma, and miR-382-5p expression may have effects on the malignancy of osteosarcoma cells. Thus, miR-382-5p expression may potentially act as a prognostic biomarker or a therapeutic target in osteosarcoma.

\section{Acknowledgements}

Not applicable.

\section{Funding}

The present study was supported by the Sichuan Science and Technology Program (grant nos. 2020YFS0529, 2019YJ0707 and 2018SZ0377), the Nanchong Science and Technology Program (grant nos. 19SXHZ0230, 19SXHZ0451, 19SXHZ0345, 18SXHZ0366 and 18SXHZ0370) and the Science and Technology Project of the Health Planning Committee of Sichuan (grant nos. 19PJ057 and 20PJ177).

\section{Availability of data and materials}

The datasets used and/or analyzed in this study are available from the corresponding author upon reasonable request.

\section{Authors' contributions}

KL and GS conceived and designed the experiments. HW, YXL, MLZ and JB performed the experiments. WH, MY, RP, NXH and GF analyzed and interpreted the data. WH and MY collected the clinical samples and performed the experiments involving the clinical samples. WH and HW drafted the initial manuscript. GS and KL revised the manuscript for important intellectual content. KL and GS confirm the authenticity of all the raw data. All authors have read and approved the final manuscript.

\section{Ethics approval and consent to participate}

The present study was approved by the Institutional Center Ethics Review Committee of Nanchong Central Hospital (Nanchong, China; approval no. 2020-002) and written informed consent was provided by all patients or their guardians prior to the study start. All animal experiments were performed in accordance with the Guide for the Care and Use of Laboratory Animals (National Academies Press, 2011; Washington, USA). The animal experimental protocol was approved by the Ethical Review Committee for Animal Experiments of Nanchong Central Hospital (Nanchong, China; approval no. NSMC-2020-42) and all experiments were performed according to the AVMA guidelines.

\section{Patient consent for publication}

Not applicable.

\section{Competing interests}

The authors declare that they have no competing interests.

\section{References}

1. Broadhead ML, Clark JC, Myers DE, Dass CR and Choong PF: The molecular pathogenesis of osteosarcoma: A review. Sarcoma 2011: 959248, 2011.

2. Yang J and Zhang W: New molecular insights into osteosarcoma targeted therapy. Curr Opin Oncol 25: 398-406, 2013.

3. Kansara M, Teng MW, Smyth MJ and Thomas DM: Translational biology of osteosarcoma. Nat Rev Cancer 14: 722-735, 2014.

4. Ottaviani G and Jaffe N: The epidemiology of osteosarcoma. Cancer Treat Res 152: 3-13, 2009.

5. Wu CC, Beird HC, Andrew Livingston J, Advani S, Mitra A, Cao S, Reuben A, Ingram D, Wang WL, Ju Z, et al: Immuno-genomic landscape of osteosarcoma. Nat Commun 11: 1008, 2020.

6. Rytting M, Pearson P, Raymond AK, Ayala A, Murray J, Yasko AW, Johnson M and Jaffe N: Osteosarcoma in preadolescent patients. Clin Orthop Relat Res: 39-50, 2000.

7. Petrilli AS, de Camargo B, Filho VO, Bruniera P, Brunetto AL Jesus-Garcia R, Camargo OP, Pena W, Péricles P, Davi A, et al: Results of the Brazilian osteosarcoma treatment group studies III and IV: Prognostic factors and impact on survival. J Clin Oncol 24: 1161-1168, 2006.

8. Hosseinahli N, Aghapour M, Duijf PHG and Baradaran B Treating cancer with microRNA replacement therapy: A literature review. J Cell Physiol 233: 5574-5588, 2018.

9. Aredia F and Scovassi AI: A new function for miRNAs as regulators of autophagy. Future Med Chem 9: 25-36, 2017.

10. Song B, Wang Y, Xi Y, Kudo K, Bruheim S, Botchkina GI, Gavin E, Wan Y, Formentini A, Kornmann M, et al: Mechanism of chemoresistance mediated by miR-140 in human osteosarcoma and colon cancer cells. Oncogene 28: 4065-4074, 2009.

11. Naeli P, Yousefi F, Ghasemi Y, Savardashtaki A and Mirzaei H: The role of MicroRNAs in lung cancer: Implications for diagnosis and therapy. Curr Mol Med 20: 90-101, 2020.

12. Martens-Uzunova ES, Bottcher R, Croce CM, Jenster G, Visakorpi T and Calin GA: Long noncoding RNA in prostate, bladder, and kidney cancer. Eur Urol 65: 1140-1151, 2014.

13. Zheng Z, Ding M, Ni J, Song D, Huang J and Wang J: miR-142 acts as a tumor suppressor in osteosarcoma cell lines by targeting Rac1. Oncol Rep 33: 1291-1299, 2015.

14. Qu Q, Chu X and Wang P: MicroRNA-195-5p suppresses osteosarcoma cell proliferation and invasion by suppressing naked cuticle homolog 1. Cell Biol Int 41: 287-295, 2017.

15. Wang Y, Ma Z, Kan P and Zhang B: The diagnostic value of serum miRNA-221-3p, miRNA-382-5p, and miRNA-4271 in ischemia stroke. J Stroke Cerebrovasc Dis 26: 1055-1060, 2017.

16. Zhang X, Zhao H, Zhang Y, Yang X, Zhang J, Yi M and Zhang C: The MicroRNA-382-5p/MXD1 axis relates to breast cancer progression and promotes cell malignant phenotypes. J Surg Res 246: 442-449, 2020.

17. Tan H, He Q, Gong G, Wang Y, Li J, Wang J, Zhu D and Wu X: miR-382 inhibits migration and invasion by targeting ROR 1 through regulating EMT in ovarian cancer. Int J Oncol 48: 181-190, 2016.

18. Shan N, Shen L, Wang J, He D and Duan C: miR-153 inhibits migration and invasion of human non-small-cell lung cancer by targeting ADAM19. Biochem Biophys Res Commun 456: 385-391, 2015.

19. Wu Z, He B, He J and Mao X: Upregulation of miR-153 promotes cell proliferation via downregulation of the PTEN tumor suppressor gene in human prostate cancer. Prostate 73: 596-604, 2013.

20. Paraskevopoulou MD, Vlachos IS, Karagkouni D, Georgakilas G, Kanellos I, Vergoulis T, Zagganas K, Tsanakas P, Floros E, Dalamagas $T$ and Hatzigeorgiou AG: DIANA-LncBase v2: Indexing microRNA targets on non-coding transcripts. Nucleic Acids Res 44 (D1): D231-D238, 2016.

21. Livak KJ and Schmittgen TD. Analysis of relative gene expression data using real-time quantitative PCR and the 2(-Delta Delta C(T)) method. Methods 25: 402-408, 2001.

22. Lee JH, Kim C, Baek SH, Ko JH, Lee SC, Yang WM, Um JY, Sethi G and Ahn KS: Capsazepine inhibits JAK/STAT3 signaling, tumor growth, and cell survival in prostate cancer. Oncotarget 8: 17700-17711, 2017.

23. Sticht C, De La Torre C, Parveen A and Gretz N: miRWalk: An online resource for prediction of microRNA binding sites. PLoS One 13: e0206239, 2018.

24. Rennie W, Kanoria S, Liu C, Mallick B, Long D, Wolenc A, Carmack CS, Lu J and Ding Y: STarMirDB: A database of microRNA binding sites. RNA Biol 13: 554-560, 2016. 
25. Zhu HF, Wang YH, Liu D, Sun XQ, and Wang FR: Vanadium rutin complex sensitizes breast cancer cells via modulation of p53/Bax/Bcl2/VEGF correlated with apoptotic events. Acta Pol Pharm Drug Res 77: 89-98, 2020.

26. Lee RC, Feinbaum RL and Ambros V: The C. elegans heterochronic gene lin-4 encodes small RNAs with antisense complementarity to lin-14. Cell 75: 843-854, 1993.

27. Nugent M: MicroRNA function and dysregulation in bone tumors: The evidence to date. Cancer Manag Res 6: 15-25, 2014

28. Li S, Fu H, Wang Y, Tie Y, Xing R, Zhu J, Sun Z, Wei L and Zheng X: MicroRNA-101 regulates expression of the $v$-fos FBJ murine osteosarcoma viral oncogene homolog (FOS) oncogene in human hepatocellular carcinoma. Hepatology 49: 1194-1202, 2009.

29. Vishnol A and Rani S: miRNA biogenesis and regulation of disease: An overview. Methods Mol Biol 1509: 1-10, 2017.

30. Reddy KB: MicroRNA (miRNA) in cancer. Cancer Cell Int 15 $38,2015$.

31. Ho JY, Hsu RJ, Liu JM, Chen SC, Liao GS, Gao HW and Yu CP MicroRNA-382-5p aggravates breast cancer progression by regulating the RERG/Ras/ERK signaling axis. Oncotarget 8: 22443-22459, 2017.

32. Wang J, Chen C, Yan X and Wang P: The role of miR-382-5p in glioma cell proliferation, migration and invasion. Onco Targets Ther 12: 4993-5002, 2019.

33. Xu M, Jin H, Xu CX, Sun B, Song ZG, Bi WZ and Wang Y: miR-382 inhibits osteosarcoma metastasis and relapse by targeting Y box-binding protein 1. Mol Ther 23: 89-98, 2015.

34. Wang L, Wang P, Su X and Zhao B: Circ_0001658 promotes the proliferation and metastasis of osteosarcoma cells via regulating miR-382-5p/YB-1 axis. Cell Biochem Funct 38: 77-86, 2020

35. Xiao Y, Li C, Wang H and Liu Y: LINC00265 targets miR-382-5p to regulate SAT1, VAV3 and angiogenesis in osteosarcoma. Aging (Albany NY) 12: 20212-20225, 2020.

36. Kobayashi E, Hornicek FJ and Duan Z: MicroRNA involvement in osteosarcoma. Sarcoma 2012: 359739, 2012

37. Bielack SS, Kempf-Bielack B, Delling G, Exner GU, Flege S, Helmke K, Kotz R, Salzer-Kuntschik M, Werner M, Winkelmann W, et al: Prognostic factors in high-grade osteosarcoma of the extremities or trunk: An analysis of 1,702 patients treated on neoadjuvant cooperative osteosarcoma study group protocols. J Clin Oncol 20: 776-790, 2002.
38. Nugent M: microRNA and bone cancer. Adv Exp Med Biol 889: 201-230, 2015

39. Wu L and Qu X: Cancer biomarker detection: Recent achievements and challegenges. Chem Soc Rev 44: 2963-2997, 2015.

40. Wang XX, Ye FG, Zhang J, Li JJ, Chen QX, Lin PY and Song CG: Serum miR-4530 sensitizes breast cancer to neoadjuvant chemotherapy by suppressing RUNX2. Cancer Manag Res 10: 4393-4400, 2018.

41. Sampson VB, Yoo S, Kumar A, Vetter NS and Kolb EA: MicroRNAs and potential targets in osteosarcoma: Review. Front Pediatr 3: 69, 2015.

42. AlAbdi L, He M, Yang Q, Norvil AB and Gowher H: The transcription factor Vezf1 represses the expression of the antiangiogenic factor Cited2 in endothelial cells. J Biol Chem 293 11109-11118, 2018

43. He M, Yang Q, Norvil AB, Sherris D and Gowher $\mathrm{H}$ : Characterization of small molecules inhibiting the pro-angiogenic activity of the zinc finger transcription factor Vezf1. Molecules 23: 1615, 2018.

44. Gerald D, Adini I, Shechter S, Prendergast GC, Klagsbrun M Stuhlmann H, Rigby AC, Nagy JA and Benjamin LE: RhoB controls coordination of adult angiogenesis and lymphangiogenesis following injury by regulating VEZF1-mediated transcription. Nat Commun 4: 2824, 2013.

45. Yin R, Guo L, Gu J, Li C and Zhang W: Over expressing miR-19b-1 suppress breast cancer growth by inhibiting tumor microenvironment induced angiogenesis. Int J Biochem Cell Biol 97: 43-51, 2018

46. Ahmed M, Lai TH, Zada S, Hwang JS, Pham TM, Yun M and Kim DR: Functional linkage of RKIP to the epithelial to mesenchymal transition and autophagy during the development of prostate cancer. Cancers (Basel) 10: 273, 2018.

This work is licensed under a Creative Commons Attribution-NonCommercial-NoDerivatives 4.0 International (CC BY-NC-ND 4.0) License. 